\title{
Distinct family of colliding gravitational waves in general relativity
}

\author{
Mustafa Halilsoy \\ Nuclear Engineering Department, King Abdulaziz University, P.O. Box 9027, Jeddah-21413, Saudi Arabia
}

(Received 19 February 1988)

\begin{abstract}
We present a new family of exact solutions for the Einstein equations that describes colliding gravitational shock waves with cross polarization. In the limit of single polarization it reduces to a family that, up to a transformation of its metric functions, is distinct from the well-known Szekeres family. Furthermore, this family of solutions does not belong to the largest family found recently by Ferrari, Ibañez, and Bruni.
\end{abstract}

\section{INTRODUCTION}

In recent years there has been revived interest in physics literature on the topic of colliding gravitational waves (CGW's) in general relativity. ${ }^{1-4}$ From the physics standpoint, among the topics discussed so far, the emergence of essential space-time singularities dominates. This distinctive singularity feature of colliding pure plane gravitational waves emerges in contrast with its cylindri$\mathrm{cal}^{5,6}$ and pure ${ }^{7-9}$ electromagnetic counterparts, for in the latter case an imperative singularity does not arise.

In this paper we shall derive a new family of CGW's with a cross (second) polarization that does not belong to the largest family found by Ferrari, Ibañez, and Bruni. ${ }^{1}$ However, our family of solutions does not have anything new to contribute to the singularity aspect in the topic of CGW's. In this sense, it does not contradict the generic singularity nature of the resulting space-time subsequent to the collision. In particular, horizons do not form around the singularities of our space-times. From a physical point of view these solutions represent colliding gravitational shock waves with various wave fronts. Impulse waves occur in the family for the special choice of our parameters.

In the limit of linear (single) polarization our family reduces to a family that is related to the well-known Szekeres $^{10}(S)$ family by a transformation of the metric components that will be described in the paper.

Our method of solution is to parametrize the Ernst function in terms of the solutions of the Euler-Darboux equation in the geometry of CGW's. A similar method was used in general relativity long ago, ${ }^{11}$ to integrate stationary axially symmetric Einstein fields of isolated masses. However, because of the lack of physical significance, the solutions obtained by such a method in the latter case were discarded completely. In colliding electromagnetic shock waves this method led to an interesting solution, ${ }^{9}$ and being prompted from that solution, we apply the same technique to the collision of pure gravitational waves.

In Sec. II we explain our formalism and reduce our equation into the standard Ernst ${ }^{12}$ form. A particular family of solutions and analysis of its physical properties follow in Sec. III. Concluding remarks in Sec. IV are fol- lowed by the Appendix in which we give a solution for CGW's in terms of a Painleve transcendent.

\section{THE FORMALISM}

The space-time line element that we adopt in this paper is the one introduced first by Szekeres: ${ }^{10,13,14}$

$$
\begin{aligned}
d s^{2}= & 2 e^{-M} d u d v-e^{-U} \\
& \times\left(e^{V} \cosh W d x^{2}+e^{-V} \cosh W d y^{2}-2 \sinh W d x d y\right),
\end{aligned}
$$

where all metric functions depend on the null coordinates $u$ and $v$ alone. From experience with the mathematics of CGW's it is well known that the metric function $U$ is fixed as a coordinate condition, $M$ is determined from the equations of quadratures, whereas $V$ and $W$ satisfy the Einstein equations

$2 V_{u v}-U_{u} V_{v}-U_{v} V_{u}=-2 \tanh W\left(V_{u} W_{v}+V_{v} W_{u}\right)$,

$2 W_{u v}-U_{u} W_{v}-U_{v} W_{u}=2 V_{u} V_{v} \sinh W \cosh W$.

In obtaining new solutions for colliding waves in general relativity, oblate- (prolate-)type coordinates proved to be useful and therefore we shall follow a similar trend by defining new coordinates $\tau$ and $\sigma$ by

$$
\begin{aligned}
& \tau=u^{n_{1} / 2}\left(1-v^{n_{2}}\right)^{1 / 2}+v^{n_{2} / 2}\left(1-u^{n_{1}}\right)^{1 / 2}, \\
& \sigma=u^{n_{1} / 2}\left(1-v^{n_{2}}\right)^{1 / 2}-v^{n_{2} / 2}\left(1-u^{n_{1}}\right)^{1 / 2},
\end{aligned}
$$

where $n_{1}$ and $n_{2}$ are arbitrary Szekeres $(S)$ parameters, such that $n_{i} \geq 2$. We would like to add that the geometry of CGW's allows us to introduce such parameters as powers of the null coordinates. Physically these replacements amount to the modification of the wave fronts considered in collision. On account of the constraints in axially symmetrical geometry, the oblate (prolate) coordinates in such geometries do not allow a similar generalization for $n_{i}>2$. Equations (2) and (3) become now, after employing the coordinate condition for $U$,

$e^{-U}=\sqrt{1-\tau^{2}} \sqrt{1-\sigma^{2}}=1-u^{n_{1}}-v^{n_{2}}$, 


$$
\begin{aligned}
& {\left[\left(1-\tau^{2}\right) V_{\tau}\right]_{\tau}-\left[\left(1-\sigma^{2}\right) V_{\sigma}\right]_{\sigma}} \\
& \quad=-2 \tanh W\left[\left(1-\tau^{2}\right) V_{\tau} W_{\tau}-\left(1-\sigma^{2}\right) V_{\sigma} W_{\sigma}\right], \\
& {\left[\left(1-\tau^{2}\right) W_{\tau}\right]_{\tau}-\left[\left(1-\sigma^{2}\right) W_{\sigma}\right]_{\sigma}} \\
& \quad=-\sinh W \cosh W\left[\left(1-\tau^{2}\right) V_{\tau}^{2}-\left(1-\sigma^{2}\right) V_{\sigma}^{2}\right] .
\end{aligned}
$$

We introduce next a pair of complex potentials $(Z, \eta)$, defined in terms of the metric functions $V$ and $W$ by

$$
Z \equiv \frac{1-\eta}{1+\eta}=\frac{e^{-V}}{\cosh W}(1+i \sinh W)
$$

Equations (6) and (7) are expressed in terms of these complex potentials as

$$
\begin{aligned}
& \begin{aligned}
&(\operatorname{Re} Z)\left\{\left[\left(1-\tau^{2}\right) Z_{\tau}\right]_{\tau}-\right. {\left.\left[\left(1-\sigma^{2}\right) Z_{\sigma}\right]_{\sigma}\right\} } \\
&=\left(1-\tau^{2}\right) Z_{\tau}^{2}-\left(1-\sigma^{2}\right) Z_{\sigma}^{2}, \\
&(\eta \bar{\eta}-1)\left\{\left[\left(1-\tau^{2}\right) \eta_{\tau}\right]_{\tau}-\left[\left(1-\sigma^{2}\right) \eta_{\sigma}\right]_{\sigma}\right\} \\
&=2 \bar{\eta}\left[\left(1-\tau^{2}\right) \eta_{\tau}^{2}-\left(1-\sigma^{2}\right) \eta_{\sigma}^{2}\right] .
\end{aligned}
\end{aligned}
$$

One readily identifies this pair of equations as the one satisfied by the Ernst potentials $\epsilon$ and $\xi$, associated with the Killing field that arises in a different context. For the sake of obtaining new solutions in CGW's, we shall exploit this analogy to a certain extent and refer to Eq. (10) as the Ernst equation.

We proceed now with the following parametrization of the complex potential $\eta$ :

$$
\eta=\Omega(X) e^{i \beta(X)}
$$

where both $\Omega$ and $\beta$ are real functions of their argument $X$, which satisfies the Euler-Darboux (ED) equation

$$
\left[\left(1-\tau^{2}\right) X_{\tau}\right]_{\tau}-\left[\left(1-\sigma^{2}\right) X_{\sigma}\right]_{\sigma}=0 .
$$

Since this equation has the same structure of the Laplace equation, there is no loss of generality in referring to its solutions as the harmonic functions. In the Appendix we parametrize $\eta$ with two independent harmonic functions and obtain results in terms of Painleve's fifth transcendent.

We recall that in the null coordinates the ED equation reads ${ }^{10}$

$$
2 X_{u v}-U_{u} X_{v}-U_{v} X_{u}=0
$$

in which $U$ is given by Eq. (5)

Substituting (11) into (10) leads to the following solutions for $\Omega$ and $\beta$ (Ref. 15)

$\Omega^{2}=\frac{\cosh 2 X-\cos \theta}{\cosh 2 X+\cos \theta}, \quad \tan \beta=-(\tan \theta) \operatorname{coth} 2 X$,

where $\theta$ is a constant of integration that will be interpreted, as in the case of colliding electromagnetic waves, ${ }^{9}$ to measure the second (cross) polarization of the waves in collision. From Eq. (8) and solution (14) we calculate the expression for $\boldsymbol{Z}$ by

$$
Z(X, \theta)=\frac{\cos \theta-i \sin \theta \cosh 2 X}{\cosh 2 X-\cos \theta \sinh 2 X} .
$$

We may employ this expression for $Z$ in Eq. (8) to find the metric functions $V$ and $W$. However, if this is done, one can show easily that by a global rotation of the $(x, y)$ coordinate axes, the polarization angle will be set to zero; thus the metric will diagonalize. To overcome this difficulty we introduce the standard Ernst potential ${ }^{12}$

$$
\epsilon=e^{2 \Psi}+i \Phi
$$

where $e^{2 \Psi}=-g_{x x}$ and $\Phi$ is a twist potential, and make use of the well-known trick in the Ernst formalism. Namely, if (16) solves the Ernst equation given by Eq. (9) then there is an associated solution given by

$$
\epsilon=e^{-U_{e}-2 \Psi}+i \omega
$$

provided the function $\omega$ is integrated from the pair of integrability equations

$$
\begin{aligned}
& \omega_{\tau}=e^{-U_{e} e^{-4 \Psi} \Phi_{\sigma}}, \\
& \omega_{\sigma}=e^{-U_{e} e^{-4 \Psi} \Phi_{\tau} .}
\end{aligned}
$$

We require now Ernst's $\epsilon$, defined in (16) to be identical with $Z$ in (15) [and therefore in (8)], since they both satisfy the same equation. This requirement expresses $\omega$ and $\Psi$ in terms of $V$ and $W$ in accordance with

$$
\omega=e^{-V} \tanh W, \quad \omega e^{2 \Psi}=e^{-U} \sinh W .
$$

When the values of $\Psi$ and $\Phi$ obtained in this way and $U$ from (5) are put into Eqs. (18) and (19), we obtain

$$
\begin{aligned}
& \omega_{\tau}=2 \tan \theta\left(1-\sigma^{2}\right) X_{\sigma}, \\
& \omega_{\sigma}=2 \tan \theta\left(1-\tau^{2}\right) X_{\tau} .
\end{aligned}
$$

Thus, the integrability equations for $\omega$ reduce to the condition for $X$ to satisfy the ED equation (12), a condition that we have already assumed $a$ priori. In the next section we shall make a particular choice for $X$ and determine the metric functions explicitly.

\section{A FAMILY OF EXACT SOLUTIONS}

While the choice for $X$ as the solution of the ED equation (12) can be much more general, at this point we shall make the choice

$$
e^{2 X}=\left(\frac{1+\tau}{1-\tau}\right)^{\left(k_{1}+k_{2}\right) / 4}\left(\frac{1+\sigma}{1-\sigma}\right)^{\left(k_{1}-k_{2}\right) / 4},
$$

due to Szekeres. ${ }^{10}$ The parameters $\left(k_{1}, k_{2}\right)$ are related to $\left(n_{1}, n_{2}\right)$, through the Einstein equations by

$$
k_{i}^{2}=8\left(1-\frac{1}{n_{i}}\right) \quad(i=1,2) .
$$

Using this value for $X$ in Eqs. (21) and (22) we obtain $\omega$ easily as

$$
\omega=\frac{1}{2} \tan \theta\left[\left(k_{1}-k_{2}\right) \tau+\left(k_{1}+k_{2}\right) \sigma\right],
$$

and the Ernst potential $\epsilon$ takes the form 


$$
\begin{aligned}
\epsilon= & \frac{1}{\cos \theta} e^{-U}\left(e^{2 X} \sin ^{2} \frac{\theta}{2}+e^{-2 X} \cos ^{2} \frac{\theta}{2}\right) \\
& +\frac{i}{2} \tan \theta\left[\left(k_{1}-k_{2}\right) \tau+\left(k_{1}+k_{2} \sigma\right) \sigma\right],
\end{aligned}
$$

in which $U$ and $X$ are given by (5) and (23), respectively. Direct substitution of this expression into Eq. (9), in place of $Z$, verifies that it is truly an Ernst solution. Also, we have the freedom to add one more parameter to our $\epsilon$ by considering its Ehlers transform. This, however, will not enhance the physics of CGW's at our disposal and there- fore we shall ignore it.

The result for the metric functions $V$ and $W$ follow directly from Eqs. (20) and (15) for $\Psi$, whereas the metric function $M$ is integrated from the equations of quadratures $^{10}$

$$
\begin{aligned}
& 2 U_{u u}-U_{u}^{2}+2 U_{u} M_{u}=W_{u}^{2}+V_{u}^{2} \cosh ^{2} W, \\
& 2 U_{v v}-U_{v}^{2}+U_{v} M_{v}=W_{v}^{2}+V_{v}^{2} \cosh ^{2} W,
\end{aligned}
$$

which are to be transformed into the $(\tau, \sigma)$ coordinates, for a straightforward calculation. The results are

$$
\begin{aligned}
& \frac{e^{-V}}{\cosh W}=\frac{e^{-U}}{\cos \theta}\left(e^{2 X} \sin ^{2} \frac{\theta}{2}+e^{-2 X} \cos ^{2} \frac{\theta}{2}\right) \\
& e^{-V} \tanh W=\frac{1}{2} \tan \theta\left[\left(k_{1}-k_{2}\right) \tau+\left(k_{1}+k_{2}\right) \sigma\right], \\
& e^{-M}=2^{\left(k_{1}^{2}+k_{2}^{2}\right) / 8}\left(1-\tau^{2}\right)^{\left(k_{1}+k_{2}\right)^{2} / 16}\left(1-\sigma^{2}\right)^{\left(k_{1}-k_{2}\right)^{2} / 16} \\
& \times\left(1+\tau \sigma+\sqrt{1-\tau^{2}} \sqrt{1-\sigma^{2}}\right)^{-k_{1}^{2} / 8}\left(1-\tau \sigma+\sqrt{1-\tau^{2}} \sqrt{1-\sigma^{2}}\right)^{-k_{2}^{2} / 8}\left(e^{2 X} \sin ^{2} \frac{\theta}{2}+e^{-2 X} \cos ^{2} \frac{\theta}{2}\right),
\end{aligned}
$$

where $U$ and $X$ are given in (5) and (23), respectively. The incoming metrics for region II and III are found by setting $v=0$ and $u=0$, respectively, and since these regions have common aspects, we shall study only region II. We obtain region II functions by substituting $\tau=\sigma=u$ :

$$
\begin{aligned}
& e^{-U}=1-u^{n_{1}}, \quad \sinh W=\left(k_{1} \sin \theta\right) \frac{u^{n_{1} / 2}\left(1-u^{n_{1}}\right)^{k_{1} / 2-1}}{\left(1+u^{n_{1}}\right)^{k_{1}} \sin ^{2} \frac{\theta}{2}+\left(1+u^{n_{1}}\right)^{k_{1}} \cos ^{2} \frac{\theta}{2}} \\
& e^{-2 V}=\frac{1}{\cos ^{2} \theta}\left(1-u^{n_{1}}\right)^{2-k_{1}}\left(\left(1+u^{n_{1}}\right)^{k_{1}} \sin ^{2} \frac{\theta}{2}+\left(1-u^{n_{1}}\right)^{k_{1}} \cos ^{2} \frac{\theta}{2}\right)^{2}+\left(k_{1} \tan \frac{\theta}{2}\right)^{2} u^{n_{1}}, \\
& e^{-M}=\left(1-u^{n_{1} / 2}\right)^{-k_{1}^{2} / 8+k_{1} / 2}\left(1+u^{n_{1} / 2}\right)^{-k_{1}^{2} / 8+k_{1} / 2} .
\end{aligned}
$$

Since $u$ here has the meaning $u H(u)$, i.e., with a Heaviside unit step function, for $u<0$ it reduces to the flat-space metric, as it should. An important point, however, is that in order to make sure that physical sources are absent on the null boundary between region I (i.e., flat space) and region II, Eq. (29) must be satisfied with $u H(u)$ in place of $u$ in the metric. We would like to remark that were we to employ $S$ parameters $n_{i}$ for $n_{l}<2$, it would result in such an ambiguity on $u=0$. For this reason we make the choice for our parameters such as to satisfy $n_{i} \geq 2$.

The general structure of the only nonvanishing Weyl scalar curvature $\Psi_{4}$ in the region II is

$$
\Psi_{4}(u)=\left\{\begin{array}{l}
\text { const } \times \delta(u)+\theta(u) F(u) \text { for } n_{1}=2, n_{2} \geq 2, n_{2}=2, n_{1} \geq 2, \\
\theta(u) G(u) \text { for } n_{1}>2, n_{2}>2,
\end{array}\right.
$$

in which $F(u)$ and $G(u)$ are both functions that characterize the incoming shock-wave profiles.

In order to discuss the $\theta=0$ limit of our solutions, we would like to review some aspects of CGW's with linear polarizations. The vacuum Einstein equations are

$$
\begin{aligned}
& U_{u v}=U_{u} U_{v}, \\
& 2 U_{u u}-U_{u}^{2}+2 U_{u} M_{u}=V_{u}^{2}, \\
& 2 U_{v v}-U_{v}^{2}+2 U_{v} M_{v}=V_{v}^{2}, \\
& 2 M_{u v}+U_{u} U_{v}=V_{u} V_{v}, \\
& 2 V_{u v}-U_{u} V_{v}-U_{v} V_{u}=0 .
\end{aligned}
$$

A large family of solutions $(U, V, M)$ to this system was given by Szekeres ${ }^{10}$ to which we shall refer as the $S$ family. One can easily verify ${ }^{16,17}$ that there is a second family ( $S^{\prime}$ family) of solutions $\left(U^{\prime}, V^{\prime}, M^{\prime}\right)$, related to the $S$ family by

$$
\begin{aligned}
& U^{\prime}=U, \quad V^{\prime}=V+a u \\
& M^{\prime}=M+a V+\frac{a^{2}}{2} U \quad(a=\text { const }) .
\end{aligned}
$$

We have discussed elsewhere ${ }^{16,17}$ that such a relation between two different solutions forms an isometry on the configuration manifold, where the dynamics of general 
relativity is expressed in the language of harmonic maps. Such isometries, however, have nothing to do with the isometries of the space-time manifold and therefore the relation between $S$ and $S^{\prime}$ families cannot be reduced to a mere coordinate transformation.

It can be checked that in the limit $\theta=0$ our solutions
(29) - (31) do not reduce to the $S$ family, but reduce to the $S^{\prime}$ family for the particular parameter $a=1$.

The particular case for $k_{1}=k_{2}=-2$ (or $n_{1}=n_{2}=2$ ) which is known as the Nutku-Halil ${ }^{13}(\mathrm{NH})$ solution in the $S$ family, corresponds in the $S^{\prime}$ family to the (for later reference we abbreviate $\mathrm{NH}^{\prime}$ ) solution

$$
\begin{aligned}
& e^{-U}=\left(1-\tau^{2}\right)^{1 / 2}\left(1-\sigma^{2}\right)^{1 / 2}, \quad e^{-M}=\frac{2\left(1-2 \tau \cos \theta+\tau^{2}\right)}{\left(1-\tau^{2}\right)^{1 / 2}+\left(1-\sigma^{2}\right)^{1 / 2}}, \\
& \sinh W=\frac{-2 \sigma\left(1-\tau^{2}\right)^{1 / 2} \sin \theta}{\left(1-\sigma^{2}\right)^{1 / 2}\left(1-2 \tau \cos \theta+\tau^{2}\right)}, \\
& e^{V}=\frac{\left(1-\tau^{2}\right)^{1 / 2} \cos \theta}{\left[\left(1-\sigma^{2}\right)\left(1-2 \tau \cos \theta+\tau^{2}\right)^{2}+4 \sigma^{2}\left(1-\tau^{2}\right) \sin ^{2} \theta\right]^{1 / 2}}
\end{aligned}
$$

where

$$
\tau=u \sqrt{1-v^{2}}+v \sqrt{1-u^{2}}, \quad \sigma=u \sqrt{1-v^{2}}-v \sqrt{1-u^{2}} .
$$

We would like to add that this solution overlaps with the family of solutions given by Ferrari, Ibañez, and Bruni, ${ }^{1}$ for their particular parameters $s_{1}=1, s_{2}=0$. This is expected, since after all, for $n_{1}=n_{2}=2$, our coordinates in this paper coincide with theirs. The region II line element of the $\mathrm{NH}^{\prime}$ solution is given by

$$
d s^{2}=2 \frac{(1-u)^{3 / 2}}{(1+u)^{1 / 2}} d u d v-\frac{1+u}{1-u} d x^{2}-(1+u)(1-u)^{3} d y^{2}
$$

which represents impulsive waves accompanied by shock waves. This is to be compared with the simplicity of the incoming metric of the Khan-Penrose ${ }^{18}$ solution

$$
d s^{2}=2 d u d v-(1+u)^{2} d x^{2}-(1-u)^{2} d y^{2}
$$

to which it is related by the transformation (35).

In order to see the singular points (hypersurfaces) in our general solution (29)-(31) we calculate the nonvanishing Weyl scalars of the Newman-Penrose formalism. From the nonvanishing $\Psi_{0}, \Psi_{4}$, and $\Psi_{2}$ the most compact one is $\Psi_{2}$ and we calculate it in the null tetrad of Szekeres ${ }^{10}$ as

$$
\begin{aligned}
& \Psi_{2}=\frac{n_{1} n_{2} u^{n_{1} / 2-1} v^{n_{2} / 2-1}}{4\left(1-u^{n_{1}}\right)^{1 / 2}\left(1-v^{n_{2}}\right)^{1 / 2}} \int\left\{\frac{3 \tau(\tau-\cos \theta)+\cos 2 \theta-\tau^{3} \cos \theta}{\left(1-2 \tau \cos \theta+\tau^{2}\right)^{2}}+\frac{\tau^{4} \sqrt{1-\sigma^{2}}-\sigma^{4} \sqrt{1-\tau^{2}}}{2 \sqrt{1-\tau^{2} \sqrt{1-\sigma^{2}}}\left(\sqrt{1-\tau^{2}}+\sqrt{1-\sigma^{2}}\right)}\right. \\
& +i \sin \theta\left[\frac{\tau}{1-\sigma^{2}}+\frac{1-\tau^{2}}{\left(1-\sigma^{2}\right)\left(1-2 \tau \cos \theta+\tau^{2}\right)^{2}+4 \sigma^{2}\left(1-\tau^{2}\right) \sin ^{2} \theta}\right. \\
& \times\left(2(\tau-\cos \theta)-\frac{\sigma^{2}}{1-\tau^{2}}\left(3 \tau-\tau^{3}-2 \cos \theta\right)\right. \\
& -\frac{4 \tau \sigma^{2} \sin ^{2} \theta}{\left(1-\sigma^{2}\right)\left(1-2 \tau \cos \theta+\tau^{2}\right)} \\
& \left.\left.\left.+\frac{4 \sigma^{2} \sin ^{2} \theta\left(3 \tau-\tau^{3}-2 \cos \theta\right)}{\left(1-2 \tau \cos \theta+\tau^{2}\right)^{2}}\right]\right)\right\} \int .
\end{aligned}
$$

We readily observe that essential singularities occur on the hypersurfaces $u=1, v=1, \tau=1$, and $\sigma=1$. The latter two surfaces are equivalent in the null coordinates to

$$
u^{n_{1}}+v^{n_{2}}=1
$$

which is the usual hypersurface of essential singularity that arise in the $S$ family. One can predict, without going into a detailed analysis, that $S$ and $S^{\prime}$ families have common singular hypersurfaces.
One interesting aspect of the $\mathrm{NH}^{\prime}$ solution (36) that is also shared by the NH solution is that the simultaneous changes $u \rightarrow-u$ and $v \rightarrow-v$ (or $\tau \rightarrow-\tau, \sigma \rightarrow-\sigma$ ) in the solution results also in the solution. This property provides us an alternative interpretation for the CGW, as follows: the singularity, $u^{2}+v^{2}=1$, which is located in the past $(t<0)$, evolves in time such that at $t=0$, outgoing gravitational waves emerge. Accordingly, NH and $\mathrm{NH}^{\prime}$ space-times decay into impulsive gravitational waves and impulsive waves accompanied with shock waves, respectively. 


\section{CONCLUDING REMARKS}

The family of solutions presented in this paper is characterized by three independent parameters: $n_{1}, n_{2}$ (or $k_{1}, k_{2}$ ), and $\theta$. Physically it describes the collision of gravitational shock waves that for the particular case $\left(n_{i}=2\right)$ contain also an impulsive term $\delta(u)$, accompanied by shock waves. Our solution shows that the largest family of solutions of Ferrari, Ibañez, and Bruni can be enlarged further by employing the $S$ parameters $n_{1}$ and $n_{2}$. In the limit $\theta=0$, our family reduces to $S^{\prime}$ family rather than $S$ family. It remains open however, to see whether $S$ and $S^{\prime}$ families with the second polarizations are transformable into each other [i.e., the generalization of the transformation (35) for $W \neq 0$ ].

Finally, we would like to add that Chandrasekhar's recent $^{19}$ generalization of the diagonal metrics can be employed in our formalism to obtain the corresponding nondiagonal metrics. For any given solution for the ED (or Laplace) equation, the problem can be reduced to the determination of $\omega$ through the Eqs. (21) and (22).

\section{ACKNOWLEDGMENTS}

I would like to thank Dr. S. Fakioglu and Dr. A. M. Saatci for their kind interest in this work.

\section{APPENDIX}

We parametrize the complex potential $\eta$ now in terms of two functions as

$$
\eta(X, Y)=\Omega(X) e^{i \beta(Y)},
$$

where $\Omega$ and $\beta$ are real functions of their respective arguments $X$ and $Y$, both satisfying the ED equation (12). Direct substitution of (A1) into Eq. (10) leads to the following pair of equations:

$$
\left(\frac{\Omega^{2}-1}{\Omega^{2}+1}\right) \frac{\Omega}{\Omega^{\prime}} \frac{\beta^{\prime \prime}}{\beta^{\prime}}=2 \frac{\left(1-\tau^{2}\right) X_{\tau} Y_{\tau}-\left(1-\sigma^{2}\right) X_{\sigma} Y_{\sigma}}{\left(1-\tau^{2}\right) X_{\tau}^{2}-\left(1-\sigma^{2}\right) X_{\sigma}^{2}},
$$

$$
\frac{2 \Omega \Omega^{\prime 2}+\left(1-\Omega^{2}\right) \Omega^{\prime \prime}}{\Omega\left(1+\Omega^{2}\right) \beta^{\prime 2}}=\frac{\left(1-\tau^{2}\right) Y_{\tau}^{2}-\left(1-\sigma^{2}\right) Y_{\sigma}^{2}}{\left(1-\tau^{2}\right) X_{\tau}^{2}-\left(1-\sigma^{2}\right) X_{\sigma}^{2}},
$$

where the primes stand for derivatives with respect to their respective arguments. For different choices of $X$ and $Y$ we obtain different solutions to the above set of differential equations. As an example we would like to make the choice

$$
e^{2 X}=\left(1-\tau^{2}\right)\left(1-\sigma^{2}\right), \quad Y=\tau \sigma
$$

and determine the resulting space-time metric functions. Substituting (A4) into (A2) and (A3) leads to

$$
\beta^{\prime \prime}=0
$$

and

$$
\Omega^{\prime \prime}+\frac{2 \Omega \Omega^{\prime 2}}{1-\Omega^{2}}=-\Omega\left(\frac{1+\Omega^{2}}{1-\Omega^{2}}\right) \beta^{\prime 2} e^{2 X},
$$

respectively. Equation (A5) trivially gives

$$
\beta=\beta_{0} Y+\gamma_{0}
$$

with $\beta_{0}$ and $\gamma_{0}$ two arbitrary constants of integration. For (A6), we make the change of variable in accordance with

$$
\Omega=\tanh (\Sigma / 4),
$$

and obtain

$$
\Sigma^{\prime \prime}+\beta_{0}^{2} e^{2 X}(\sinh \Sigma)=0,
$$

where a prime denotes $d / d X$. This equation is identified as a degenerate form of Painleve's fifth transcendent that arise also in cylindrical gravitational waves with cross polarization. ${ }^{5,20}$

The metric functions $V$ and $W$ can easily be expressed in terms of $\beta$ and $\Sigma$ as

$$
\begin{gathered}
e^{2 V}=\frac{1+\cos \beta \tanh \Sigma / 2}{1-\cos \beta \tanh \Sigma / 2}, \\
\sinh W=-\sin \beta \sinh \Sigma / 2 .
\end{gathered}
$$

The integration of $M$ follows from the integrability Eqs. (28) and (29) and the result is

$$
M=\frac{1}{2} X-\frac{1}{8} \int^{X} d X\left[\Sigma^{\prime 2}+4 \beta_{0}^{2} e^{2 X} \sinh ^{2} \frac{\Sigma}{2}\right]
$$

and the space-time metric components become completed.

The interesting thing in employing two independent harmonic functions $X$ and $Y$ is that the metric cannot be diagonalized so that, unlike the case treated in the paper, we do not need to integrate the metric functions $V$ and $W$ from the twist potential. We remark finally that the method used in this appendix to obtain transcendental solutions was described before. ${ }^{21} \mathrm{~A}$ detailed analysis of such transcendental functions within the context of CGW's, provided any physical interpretation can be attributed to them at all, may be the subject of a future article.
1V. Ferrari, J. Ibañez, and M. Bruni, Phys. Rev. D 36, 1053 (1987).

2V. Ferrari and J. Ibañez, Gen. Relativ. Gravit. 19, 383 (1987).

${ }^{3}$ S. Chandrasekhar and B. C. Xanthopoulos, Proc. R. Soc. London A408, 175 (1986).
${ }^{4}$ U. Yurtsever, Phys. Rev. D 36, 1662 (1987).

${ }^{5}$ S. Chandrasekhar, Proc. R. Soc. London A408, 209 (1986).

${ }^{6} \mathrm{M}$. Halilsoy (unpublished).

${ }^{7}$ P. Bell and P. Szekeres, Gen. Relativ. Gravit. 5, 275 (1974).

${ }^{8}$ S. Chandrasekhar and B. C. Xanthopoulos, Proc. R. Soc. Lon- 
don A410, 311 (1987).

${ }^{9}$ M. Halilsoy, Phys. Rev. D 37, 2121 (1988).

${ }^{10}$ P. Szekeres, J. Math. Phys. (N.Y.) 13, 286 (1972).

${ }^{11}$ A. Papapetrou, Ann. Phys. (Leipzig) 12, 309 (1953).

${ }^{12}$ F. J. Ernst, Phys. Rev. 167, 1175 (1968).

${ }^{13}$ Y. Nutku and M. Halil, Phys. Rev. Lett. 39, 1379 (1977).

${ }^{14}$ M. Halil, J. Math. Phys. (N.Y.) 20, 120 (1979).
${ }^{15}$ M. Halilsoy, Lett. Nuovo Cimento 37, 231 (1983).

${ }^{16}$ M. Halilsoy, Phys. Lett. 84A, 404 (1981).

${ }^{17}$ M. Halilsoy, J. Math. Phys. (N.Y.) 29, 320 (1988).

${ }^{18}$ K. A. Khan and R. Penrose, Nature (London) 229, 185 (1971).

${ }^{19}$ S. Chandrasekhar, Proc. R. Soc. London A415, 329 (1988).

${ }^{20} \mathrm{M}$. Halilsoy (unpublished).

${ }^{21}$ M. Halilsoy, Lett. Nuovo Cimento 44, 88 (1985). 